\title{
SPECTRAL CHARACTERIZATION OF A CLOSED CANOPY AND OPEN CANOPY FOREST IN NORTHERN SIERRA MADRE NATURAL PARK
}

\author{
C. Vidad ${ }^{1}$, C. J. Sarmiento ${ }^{2}$, C. Arellano ${ }^{1}$, R. Faelga ${ }^{1}$, R. Lopez $^{1}$, A. Maralit ${ }^{1}$, F. Pamittan ${ }^{1}$, F. Tandoc ${ }^{1}$, E. Paringit ${ }^{3}$ \\ ${ }^{1}$ Training Centre for Applied Geodesy and Photogrammetry, National Engineering Centre, University of the Philippines, Diliman \\ ${ }^{2}$ Department of Geodetic Engineering, College of Engineering, University of the Philippines, Diliman \\ ${ }^{3}$ Philippine Council for Industry, Energy and Emerging Technology Research and Development, Department of Science and \\ Technology
}

\section{Commission IV}

KEY WORDS: Spectral Mixture Analysis, Hyperspectral Imagery, Multiple Endmember Spectral Mixture Analysis

\begin{abstract}
:
Forest lands play crucial roles in nutrient recycling and climate regulation. The change of closed canopy forests to open canopy forests may indicate disturbance within the closed canopy forest. Within the local context of the Philippines, few studies have been conducted to monitor changes in closed canopy forest lands. Efforts to do so are limited by the spatial extent, remoteness and ruggedness of closed canopy forests. Satellite imagery can cover the spatial extent of forest lands as well as provide constant revisit periods for monitoring. However, while multispectral imaging can detect changes in land cover, it has limitations when detecting the subtler change from closed canopy to open canopy forest cover. This study aims to provide baseline spectral characterization of a closed canopy forest in the Philippines. For this study, a hyperspectral sensor (EO1-Hyperion) with 198 band channels ranging from $426.82 \mathrm{~nm}$ to $2395.50 \mathrm{~nm}$ and a pixel size of 30m was used to characterize the spectral variations of closed canopy forest, open canopy forest, shrubs and cropland in Northern Sierra Madre, Philippines. Multiple endmember spectral mixture analysis (MESMA) was employed to sort the image into classes as well as to characterize intra-spectral variations among the identified classes. Spectral library endmembers were assembled, optimized and used to classify the image. The spectral libraries were optimized by using Endmember Average Root Mean Square Error (EAR), Minimum Average Spectral Angle (MASA) and Iterative Endmember Selection (IES). Results overall agreement is 0.56 for EAR and IES and kappa coefficient is at 0.4.
\end{abstract}

\section{INTRODUCTION}

Philippines is located in a biodiversity hotspot with two-thirds of the earth's biodiversity (Convention on Biological Diversity, n.d.). As a result of several decades of deforestation, conversion of forest into agricultural lands and expansion of urban communities, forest coverage in the Philippines is drastically reduced from 12 million hectares in 1960 to 7 million hectares in 2015 (Food and Agricultural Organization, 2015). Efforts to conserve and protect forest lands were crafted into policies (Presidential Decree No. 389). Ensuring that these policies are taking effect on forest conservation require regular monitoring of forested lands. Monitoring the impact of these policies on the forest cover require mobilization of resources on the ground level. To solve this issue, remote sensing is employed to adequately monitor forest resource over space and time.

Remote sensing is able to provide an alternative to monitor the extent of forest cover due to its wide areal coverage and regular data acquisition. Multispectral imagery are the most common satellite image products derived from earth observation. Most earth observation sensors are open access and with data collection that had started since the 1970s. Time series imageries are useful tools in detecting and quantifying the changes on the earth's surface over time. This allows the large scale monitoring of forest cover as well as identifying deforested and degraded areas (Bullock, et.al., 2018).

The Philippines currently has an estimate of 2.028 million hectares of closed canopy forest (Forest Management Bureau, 2019). Ensuring that the remaining closed forests in the country remain intact requires not just monitoring the forest for deforestation but also for forest degradation. Forest degradation refers to "changes within the forest, whether natural or humaninduced that negatively affect the structure or function of the stand or site and thereby lower the capacity of the resulting degraded forest to supply products and/or services' (Food and Agricultural Organization, 2006). Often, degradation is not detected through the decrease of forested area but through biomass reduction and changes in species composition.

The changes required to detect forest degradation are subtler than that of deforestation, also changes indicating degradation are more specific. Main approaches used to detect degradation is through change detection in proxies and quantification of change in above ground biomass. Proxies of forest degradation include forest canopy gaps, small clearings and logging roads (Mitchell, et. al., 2017). Classification methods between closed (intact) forests and degraded forests rely on the varying spectral response of the two forests. In insular Southeast Asia, all forest types have generally closed canopy in their undisturbed state. This enables detection of canopy clearings through analysis of its spectral composition. (Miettinen, et. al., 2014).

The use of spectral mixture analysis was applied in mapping of degradation in the Amazon (Souza, 2003). The same could be applied for the forests in the Philippines despite of different drivers of forest degradation between Southeast Asia and South America (Deutsche Gesellschaft für Internationale Zusammenarbeit, 2012). This purpose of this study is to establish a baseline classification between a closed canopy forest and open canopy forest in Northern Sierra Madre in terms of their spectral characteristics. The approach used in this study is multiple endmember spectral mixture analysis which is a variant of spectral mixture analysis.

\section{MATERIALS AND METHODS}

To characterize the spectral endmembers of primary land covers, hyperspectral image from EO-1 Hyperion was used. In order to 
classify the image, a spectral library is required. This was generated by selecting the purest pixels from regions of interest where reference data is available. The computing efficiency of MESMA decreases with larger spectral library population. It is therefore necessary that a spectral library is optimized that it contains sufficient spectra that represents ground spectral variation while also maintaining computing efficiency.

\subsection{Study Area and Data Sets}

The study area is part of the Northern Sierra Madre Natural Park (NSMNP). The NSMNP was established under the Presidential Proclamation Number 978 declaring the area as a Natural Park and thus the conservations of its ecological status. The park is noted for a high species index of flora and fauna with 1079 species of trees and 638 species of animals (Biodiversity Management Bureau, 2015).

The region of interest is located in Palanan, Isabela (midpoint = $\left.17^{\circ} 59.0^{\prime} \mathrm{N}, 122^{\circ} 22.08^{\prime} \mathrm{E}\right)$. The climate in the study area is classified as tropical rainforest climate based on Köppen climate classification. Rainfall is observed at all months of the year with majority of it received on October - December. Average annual rainfall for the study area is $2610 \mathrm{~mm}$.

The hyperspectral image used in the study was acquired on February 19, 2016 by the Hyperion Sensor of EO-1 satellite. The sensor is equipped with 224 channels from $357-2576 \mathrm{~nm}$ with spectral resolution of $10 \mathrm{~nm} .70$ channels are in the Visible Near Infrared Region and 154 channels are in the Short Wave Infrared Region. The spatial resolution of the sensor is $30 \mathrm{~m}$. Hyperion imagery has a standard scene of $7.7 \mathrm{~km} \mathrm{X} 98.7 \mathrm{~km}$. A land cover map produced by the National Mapping and Resource Information Agency (NAMRIA) in 2015 was used as a reference for land cover elements.

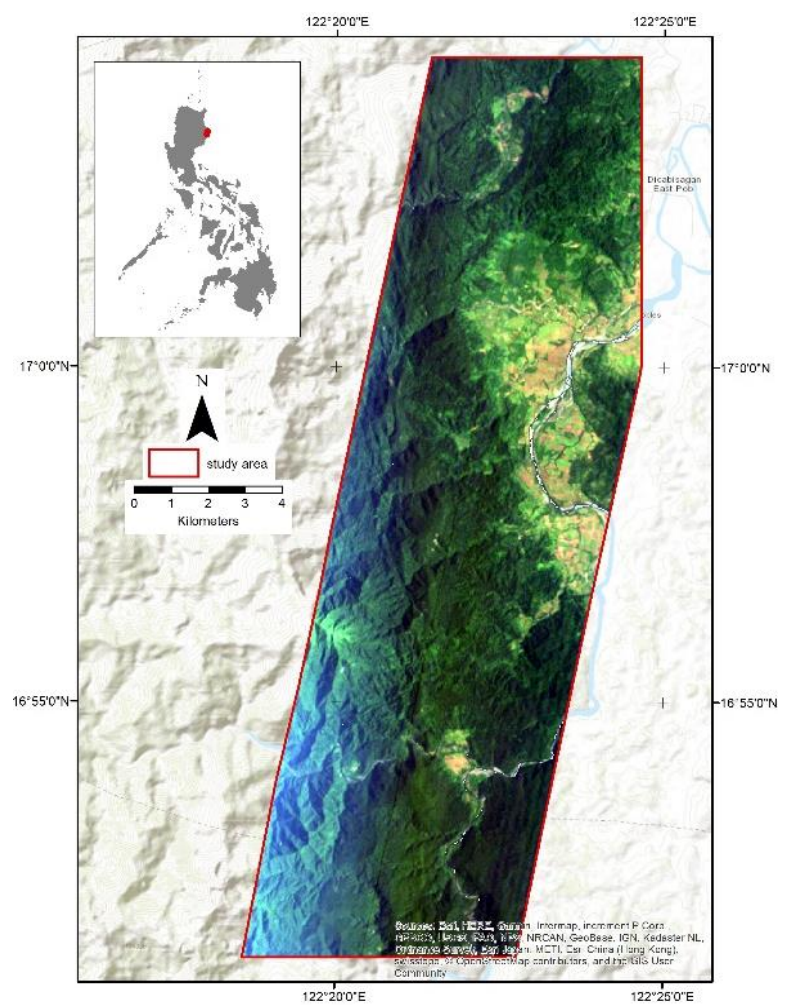

Figure 1. (Inset) Location map of the study area in Palanan, Isabela. True color composite of Hyperion image (Band 24, Band 14, Band 5)
Pre-processing of the image involves selection of bands to maximize spectral separability, destriping, application of atmospheric correction and noise removal. Table 1 provides the bands used in image processing. Fast Line-of-sight Atmospheric Analysis of Hypercubes (FLAASH) radiative transfer model was used to correct for atmospheric interferences before the image was normalized to a scale factor of 1 . A noise reduction algorithm was applied into the image using Minimum Noise Fraction (MNF) transformation.

\begin{tabular}{|c|c|}
\hline Bands & Wavelength (nm) \\
\hline $8-57$ & $426.8-925.4$ \\
\hline $79-119$ & $1195.0-1336.2$ \\
\hline $133-164$ & $1477.4-1790.2$ \\
\hline $183-184$ & $1981.9-1992.0$ \\
\hline $188-220$ & $2032.4-2355.2$ \\
\hline
\end{tabular}

Table 1. Bands of Hyperion used in image processing

MNF transformation is a two-step process, the initial step calculates the noise statistics based on per-pixel variance. The second step performs a principal component analysis that ranks the noise equivalent radiance of the image components (van der Meer, 1999). Manual inspection shows that the first 19 components can be correlated with ground features while the rest simply show increasing noise levels. MNF bands showing spatial coherence were projected back into the image space.

Finally water pixels and pixels dominated by shade were masked. Because the image was taken in a mountainous area and acquired when sun elevation is not at its highest, shadows were cast in the image. Water pixels and shadow pixels were identified using the spectra from training pixels and subsequently masked from further image processing.

\subsection{Endmember extraction}

Spectral mixture analysis (SMA) methods rely on endmember libraries to classify an image which makes the selection of possible image endmembers crucial in the process of spectral mixture analysis (Tompkins, et. al., 1997; Somers, et. al., 2011). Several algorithms have been created to select possible endmembers in a given image, one of these is the pixel purity index (PPI). Given an image composed of pure and mixed pixels, it is assumed that the spectra of mixed pixels is the result of the combination of the spectra from the pure pixels. This assumption follows that all spectra in the image are derivatives of the spectra of the purest pixels and endmembers can be represented by the spectra of purest pixels.

PPI works by plotting the radiance of pixels onto a random unit vector. Extreme pixels are determined every time it is projected onto a random unit vector, the PPI is determined by the number of time the pixel was projected. Pixels with the highest PPI are determined as the purest and represents the spectra of the endmembers of the image. This can be visualized through an $\mathrm{n}$ dimensional data cloud with the most extreme pixels plotted at the outermost edges of the cloud. The pixels defining the corners of the cloud represent the endmembers of the image (Plaza, et. al., 2004) 
The spectral library was obtained by applying PPI on the MNFtransformed image. The classes of endmembers were derived from the NAMRIA land cover map. The hyperspectral image was clipped based on the land cover class. The PPI algorithm was applied on the clipped image to obtain the probable endmembers. This was repeated for all the land cover classes in the map. This method yielded four endmember classes with 178 endmember spectra.

\subsection{Optimization of the spectral library}

Spectral Mixture Analysis (SMA) relies on the underlying assumption that the observed pixel spectra is dependent upon the abundance of image endmembers. This relationship is represented by the equation:

$$
R_{i=} \sum_{j=1}^{N} f_{j} R e_{i j}+\varepsilon_{i}
$$

Where $R_{i}$ is the observed spectral reflectance in a pixel location $i$ in an image with $n$ endmembers, $f_{j}$ is the fractional abundance of an endmember constrained under the value of $1, R e_{i j}$ is the spectral reflectance of the endmember and $\varepsilon_{i}$ is a residual.

SMA operates by assigning a single spectra to represent an endmember. Each pixel is unmixed using the spectra of extracted endmembers. A significant limitation of this method is that a land cover class can be only represented by a single endmember spectra which provides little spectral variation to the possible classification of a pixel. This can be resolved by increasing the number of possible endmembers that represent the surface material in the spectral library, however, too many endmembers used in SMA will increase the sensitivity of the method towards instrumental noise and atmospheric interference (Dennison \& Roberts, 2003)

MESMA differs from SMA by being more flexible in its classification by allowing within class-spectral variability (Roberts, et al., 1994). MESMA creates a spectral mixture model for each pixel using pre-set number of endmembers and retains the model with best for each pixel. This approach allows the possibility of multiple endmembers representing a land cover class and allowing more spectral variation in the classification of a pixel.

Following the development of a spectral library is the optimization of the number of its endmembers and which endmembers should represent a land cover class. To assess whether an endmember spectra should be used in the unmixing of the image, its Root Mean Square Error from modelling all other endmember spectra is derived using the formula:

$$
\operatorname{RMSE}=\sqrt{\frac{\sum_{\lambda=1}^{M}\left(\varepsilon_{\lambda}\right)^{2}}{M}}
$$

Where $\mathrm{M}$ is the number of bands. Linear models using different endmembers are iteratively computed as well as their RMSE. To represent the RMSE of the endmember spectra used to model all other RMSEs, a square array is created. The square array is an image composed of $n$ by $n$ pixels where $n$ is the number of endmember spectra in the library. In the square array image, the rows correspond to the endmember spectra that was used to model all the other spectra in the column. This method of representation is used to prune the spectral library and select the optimal endmembers that can classify the image.
Several methods have been developed to prune the spectral library. One of these is Endmember Average RMSE (EAR) developed by Dennison and Roberts (2003). EAR is derived for each endmember spectra by averaging the RMSE of all the models by a single endmember belonging to the same land cover class. The EAR of an endmember spectra modelling all the other spectra of a land cover class is:

$$
\mathrm{EAR}_{\mathrm{i}, \mathrm{k}}=\sum_{j=1}^{n} \frac{R M S E_{i, k}}{n-1}
$$

Where $i$ is a land cover class, $k$ is the endmember spectra of the land cover class modelling all other spectra and $n$ is the number of endmembers in the land cover class.

Minimum Average Spectral Angle (MASA) is another method to optimize the spectral library. MASA relies on spectral angle mapper (SAM) which determines the similarity between two spectra by considering each spectrum as a vector (Kruse et al., 1993). Vectors are plotted in space with a dimensionality equal to the number of bands present. The spectral similarity between two spectra is determined by calculating the angle between the two vectors where smaller angles represent higher similarity. MASA is computed as:

$$
\operatorname{MASA}_{\mathrm{i}}=\sum_{j=1}^{N} \frac{\theta_{i j}}{n-1}
$$

Where $i$ is the endmember, $j$ is the endmember that was modelled, $N$ is the number of endmembers in a landcover class, $n$ is the number of modelled spectra. $\theta$ is calculated as:

$$
\theta=\cos ^{-1}\left(\frac{\sum_{\lambda=1}^{M} \rho_{\lambda} \rho_{\lambda}^{\prime}}{L_{\rho} L_{\rho^{\prime}}}\right)
$$

Where $\rho_{\lambda}$ is the reflectance of an endmember, $\rho_{\lambda}^{\prime}$ is the reflectance of the modelled spectrum, $L_{\rho}$ is the length of an endmember vector and $L_{\rho^{\prime}}$ is the length of the endmember vector of the modelled spectrum.

The final method used to optimize the spectral library is the Iterative Endmember Selection (IES) method. IES is a semiautomatic approach that identifies a subset within a spectral library that gives the best class separability (Roth et al., 2015). The method adapted here is a step-wise procedure where IES adds or subtracts endmember spectra from the library and tests whether adding or removing an endmember improves the kappa coefficient. It iteratively goes through this process until the kappa coefficient no longer improves.

\section{RESULTS}

\subsection{Endmember Spectral library}

The results of PPI generated a spectral library grouped according to surface cover classes: closed forest, open forest, cropland, shrub and water. As seen in Figure 2, the spectra of endmembers in a cover class is variable. Spectral variability in a land cover class is of particular interest specially because land cover is rarely homogenous and its overall spectra is the product of mixing between its homogenous components. Closed forest, open forest, cropland and shrub all show vegetation endmembers, also incorporated are likely the spectra of non-photosynthetic vegetation, litter, and bare soil. 


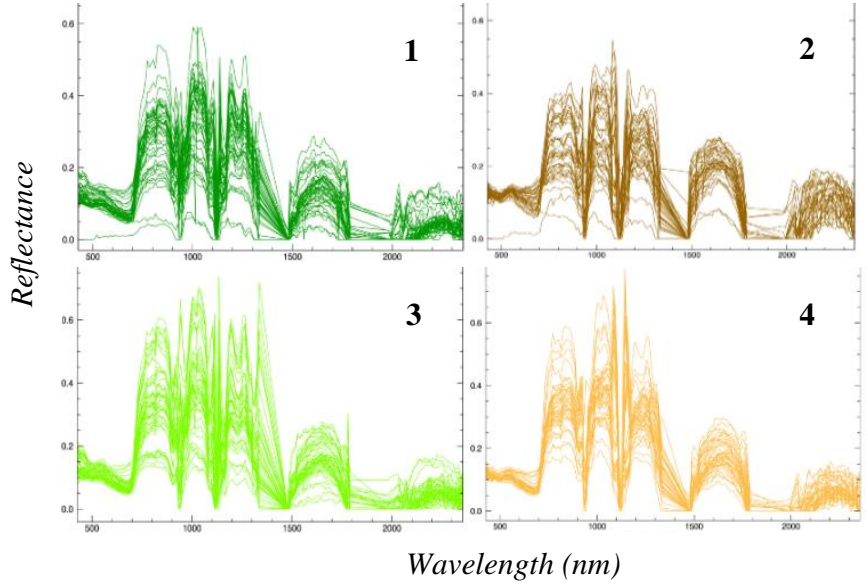

Figure 2. Results of PPI per land cover class showing the endmembers of (1) Closed forest, (2) Crop, (3) Open forest, and (4) Shrub

The square array was created to help in the optimization of the number of endmembers in the spectral library while also preserving spectral variability. A square array containing 5 bands representing RMSE, spectral angle, endmember fraction, shadow fraction, and constraints provides an indication how well endmembers model the spectra of other endmembers. The modelling of the endmember fractions was constrained between 0 and 1 , when endmember fractions do not satisfy the threshold criteria, they default to the closer threshold value. 0 value is represented by dark pixels while value of 1 is represented by bright pixels.
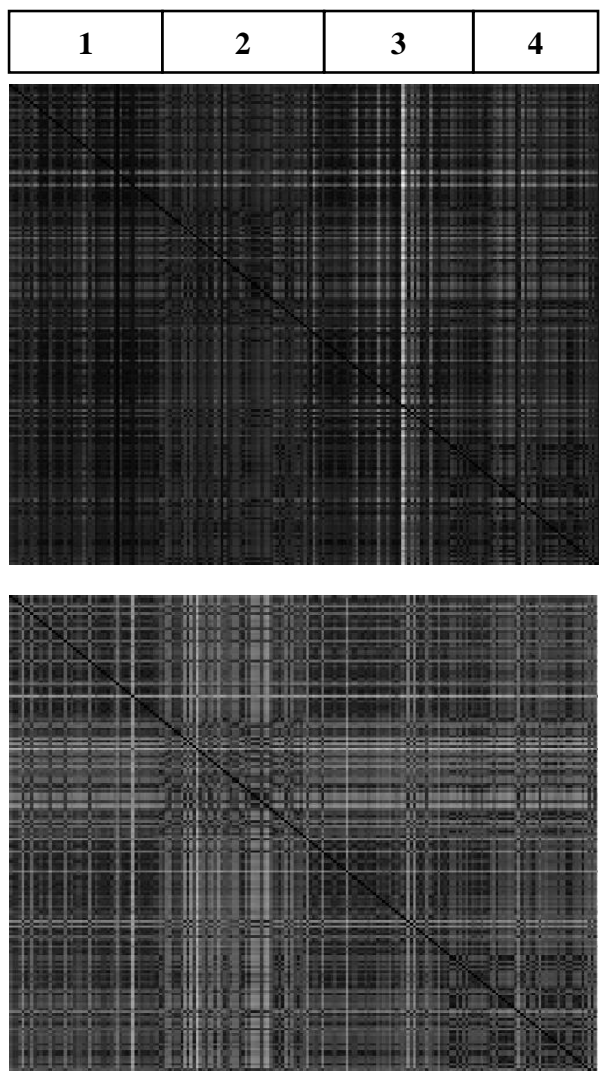

Figure 3. (Top)RMSE band of square array image; (Bottom) spectral angle band of square array image

$\begin{array}{ll}\text { 1-Closed forest } & \text { 3-Open forest } \\ \text { 2-Crop } & 4 \text {-Shrub }\end{array}$

Figure 3 shows the square array bands of RMSE and spectral angle. The clear diagonal line cutting from the upper left towards the lower right corners of the images are marked 0 to indicate that an endmember can't model itself. The top row represents endmember spectra while columns underneath represent the resulting RMSE and Spectral Angle when it modelled another endmember spectra. Spectral angle values are between 0 and $100 \%$ and the model was partially constrained to fit within the RMSE. Numbers in the boxes represent a specific land cover as shown by the image legend.

The square array indicates some endmember spectra model other endmember spectra better than others. Endmember spectra from crop land cover class show higher spectral angle values when they model the spectra of other land cover classes. This can be attributed to the abundance of bare soil spectra present in crop land cover class. A particular endmember from open forest class shows high RMSE and spectral angle value, even when modelling its own class. Spectra such as these is likely to come from an anomalous pixel that was not filtered during masking of the image.

\subsection{Optimization of the Spectral Library}

\begin{tabular}{|l|c|c|c|}
\hline Cover class & EAR & MASA & IES \\
\hline Closed forest & 34 & 33 & 15 \\
\hline Crop & 33 & 30 & 23 \\
\hline Open forest & 34 & 38 & 15 \\
\hline Shrub & 33 & 30 & 13 \\
\hline TOTAL & 134 & 131 & 66 \\
\hline
\end{tabular}

Table 2. Number of endmembers in a cover class for each spectral library

\begin{tabular}{|c|c|c|c|c|}
\cline { 2 - 5 } \multicolumn{1}{c|}{} & \multicolumn{2}{c|}{ Mean } & \multicolumn{2}{c|}{ Stdev } \\
\hline $\begin{array}{c}\text { Land cover } \\
\text { class }\end{array}$ & EAR & MASA & EAR & MASA \\
\hline $\begin{array}{c}\text { Closed } \\
\text { forest }\end{array}$ & 0.056 & 0.286 & 0.027 & 0.096 \\
\hline Crop & 0.067 & 0.358 & 0.017 & 0.060 \\
\hline Open forest & $7.2 \mathrm{E}-05$ & 0.295 & $2.5 \mathrm{E}-05$ & 0.100 \\
\hline Shrub & 0.066 & 0.288 & 0.014 & 0.045 \\
\hline
\end{tabular}

Table 3. Mean and standard deviation values in a cover class per EAR and MASA

Table 2 shows the number of endmembers for each particular cover class that was optimized using EAR, MASA and IES while Table 3 shows the mean and standard deviation of EAR and MASA for each land cover class.

\section{Optimization using supervised method}

Optimization with a supervised method where the endmember spectra belonging to a land cover class was assessed based on its EAR and MASA values. Low EAR and MASA values generally indicates a good fit of spectral similarity with other endmember spectra of the same class while higher values indicate higher spectral difference. The endmembers were chosen by setting a threshold where values that fall out of one standard deviation from the mean were excluded from the spectral library. The endmember spectra retained in the library were the likeliest endmembers to represent spectral variability but also retain a certain degree of class similarity. The EAR spectral library has a 
total of 134 endmembers while the MASA spectral library has a total of 131 endmembers.

Mean EAR in closed forest, crop and shrub cover classes have slight difference in their mean values at 0.010 whereas the open forest mean EAR has a very small value and does not fall within the range of the other land cover classes. Mean values of MASA for all land cover classes show less diversity in its range of values. The standard deviation of EAR values among land classes show that there is highest deviation from the mean in closed forest land cover while open forest shows highest deviation of MASA values.

\section{Optimization using semi-automatic method}

Another spectral library was generated by a semi-automatic method. Optimization using IES has generated a spectral library with 66 endmembers that were identified to represent the best class separability. The kappa coefficient of the final confusion matrix after an iterated procedure of adding and subtracting endmembers to improve the accuracy coefficient is 0.87 .

\subsection{Multiple Endmember Spectral Mixture Analysis}

Using the EAR, MASA and IES library, MESMA was applied on the hyperspectral image. EAR and IES spectral libraries were able to classify $96 \%$ and $92 \%$ respectively of the hyperspectral image into land cover classes while the MASA library was able to classify $7 \%$ of the image.

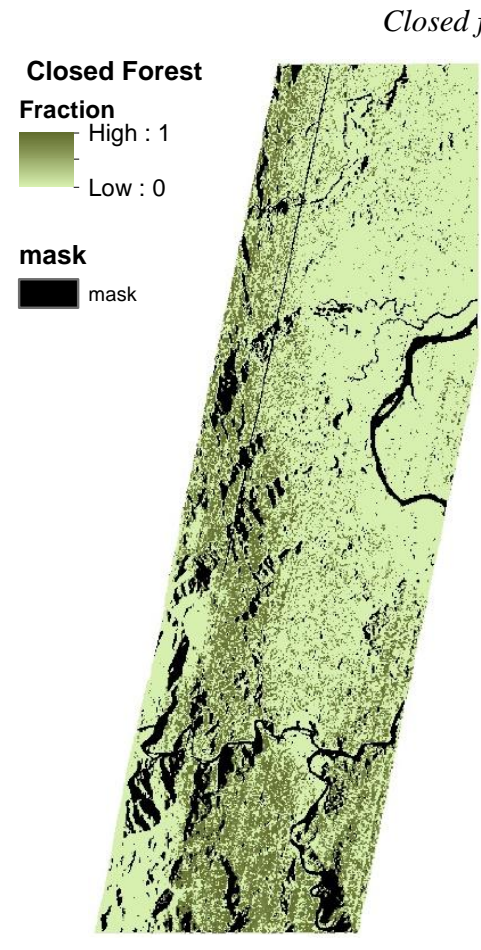

EAR

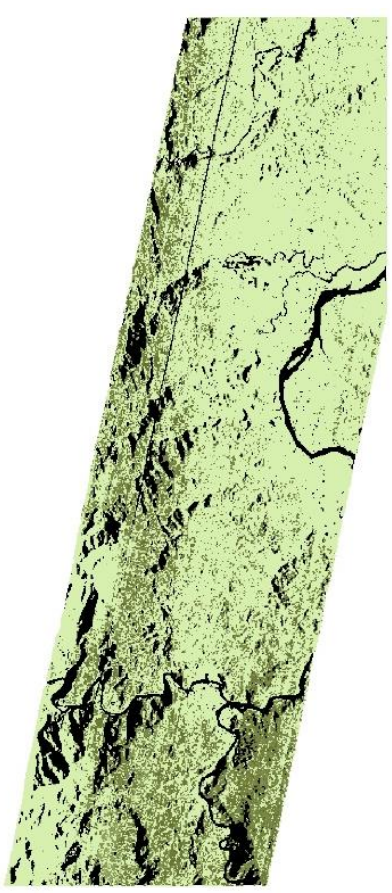

IES
Figure 4. Fractional cover of closed forest based on MESMA unmixing using EAR and IES spectral libraries

The results of MESMA unmixing of closed forests using EAR and IES spectral libraries are shown in Figure 4. Black values are the masked shadow and water pixels. Pixels with low fractional abundance of closed forests are in lighter green color while pixels with high fractional abundance of closed forests are in dark green color. For EAR, 39\% of the pixels in the image is classified as closed forest while for IES, $30 \%$ can be classified as closed forest.

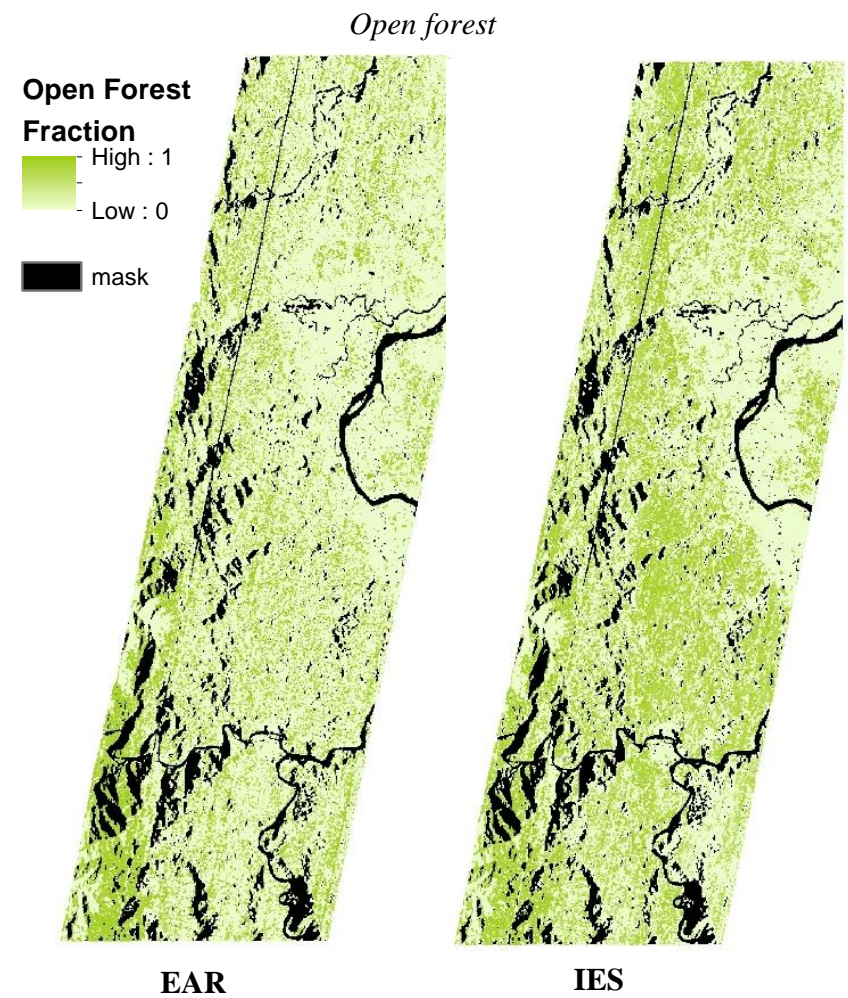

Figure 5. Fractional cover of open forest based on MESMA unmixing using EAR and IES spectral libraries

The distribution of pixels classified as open forests is presented in Figure 5. Based on the EAR library, $16 \%$ of the image is identified as open forest while the IES library identifies $38 \%$ of the image as open forest.

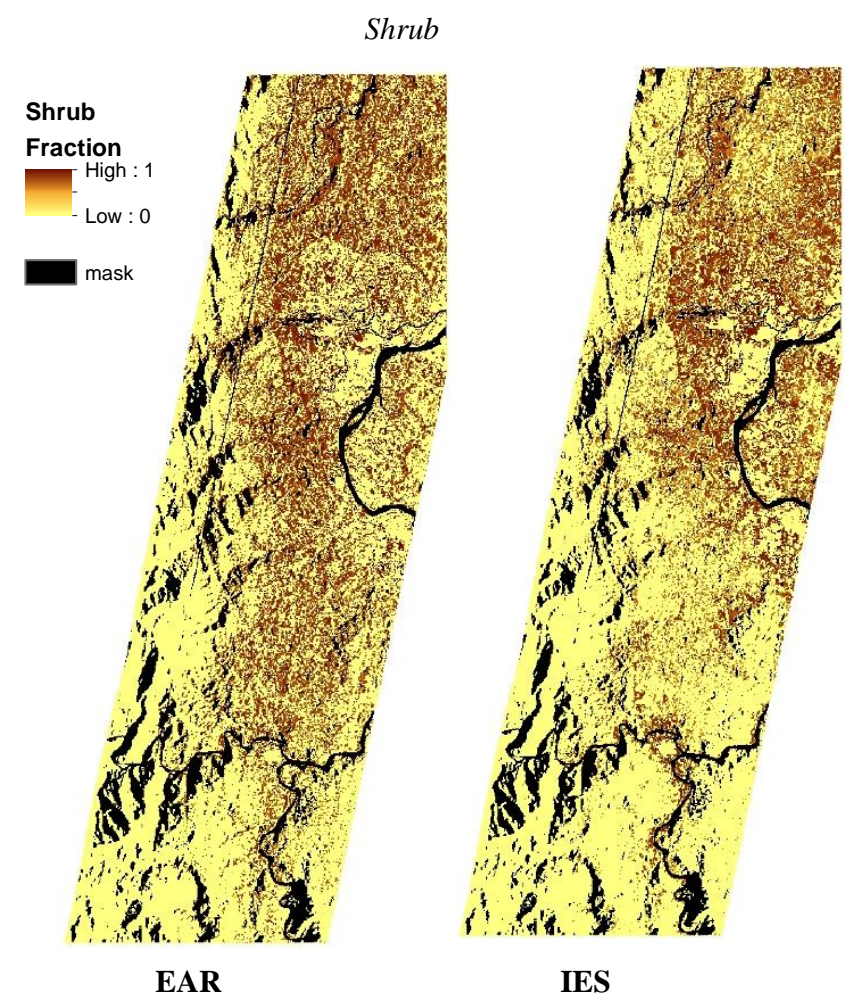

Figure 6. Fractional cover of shrubs based on MESMA unmixing using EAR and IES libraries 


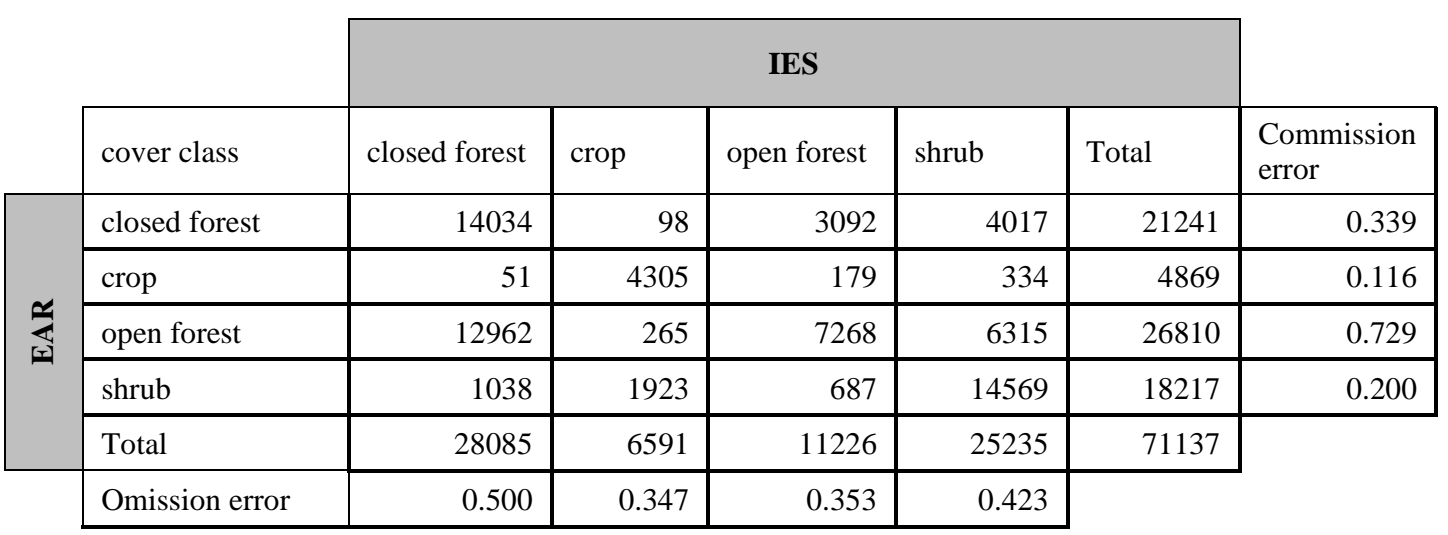

Table 4. Confusion matrix of EAR and IES classifications

Pixels classified as shrubs are shown in Figure 6. The EAR library identifies $35 \%$ of the image as shrubs while the IES library identifies $26 \%$.

Crop land cover consists of agricultural vegetation and bare surfaces under fallow. Based on classification using EAR, they cover $7 \%$ of the image while IES classified $9 \%$ of the image as crop.

\section{DISCUSSION}

EAR and IES generally provides a reasonable result of image classification of a highly vegetated area. MASA however was unable to classify the majority of the image. A comparison of EAR and MASA metrics by Dennison, et. al. in 2004 concluded that for models with low albedo variability, EAR and MASA should produce equivalent results. Shadows are cast in the image specially where there are west-dipping slopes, producing significant albedo difference in the image output and affecting the performance of MASA library in the classification of the image.

A confusion matrix in Table 5 was generated to compare the classification of EAR and IES and to measure their agreement with each other. Overall accuracy of the classification is 0.56 , the expected accuracy is 0.27 while its kappa coefficient is 0.4 . In the IES classified image, $88 \%$ of the pixels identified as crop agree with the classification of EAR. In the EAR classified image, $65 \%$ of pixels identified as crop agree with IES while $29 \%$ of the cropidentified pixels by EAR were classified as shrub by IES.

In the IES classified image, $66 \%$ of closed forest pixels are also identified likewise by EAR. For EAR classified image, $50 \%$ of closed forest pixels share the same classification with IES and $46 \%$ of closed forest pixels of EAR are identified by IES as open forest. Agreement with open forest shows that for IES, $27 \%$ of the class agree with EAR while in the EAR classified image, $65 \%$ agrees with IES classification. IES also identified more open forest pixels than EAR and there is low agreement in their classification of a closed forest and an open forest i.e., what is an open forest to IES is classified by EAR as a closed forest. IES classified image identified $80 \%$ of pixels identified as shrubs agree with the identification of EAR while for EAR, 58\% of its shrub pixels concur with IES. Figure 7 shows examples of disparity in the classification of EAR and IES.

Selection of endmember spectra that is included in the spectral library is a crucial point. The effect of endmember selection propagates in the method. In this case, pixel purity was used to select endmember spectra from which three libraries were created

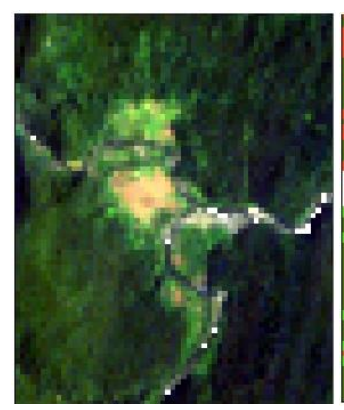

TCC

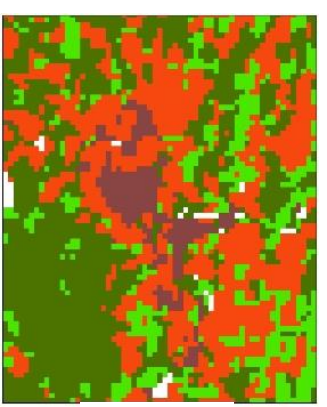

EAR

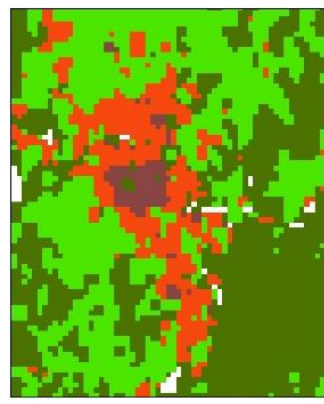

IES

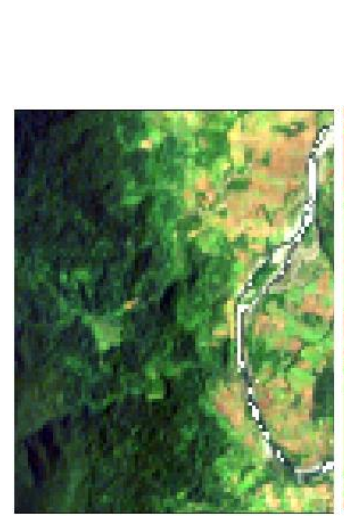

TCC

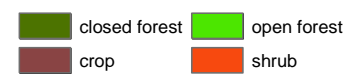

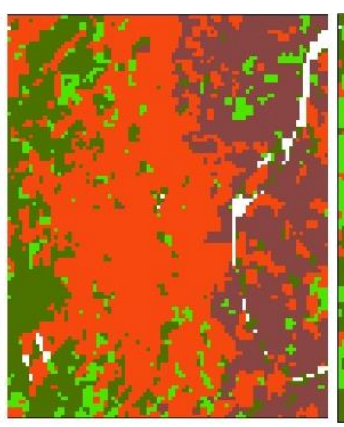

EAR

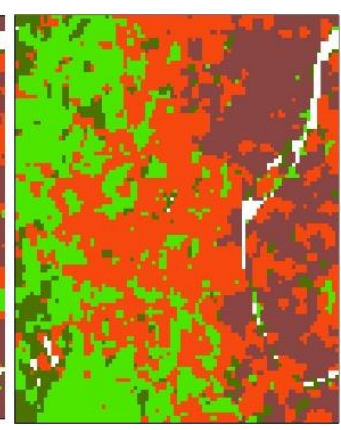

IES

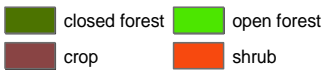

Figure 7. Comparison of true color composite and classification of the image using EAR and IES libraries

and used to classify the image.

EAR and IES show highest disagreement when classifying closed forests and open forests. This may be attributed to similarity of the endmember spectra between closed and open forest (Figure 2). Theoretically, spectra that is collected from a closed forest is dominated by reflectance from tree canopies and the reflectance from litter, bare ground and non-photosynthetic vegetation are minimal. Open forest spectra are influenced more by non-photosynthetic vegetation, litter and bare surface. The mapping of forest degradation by Souza (2003) relied on the thresholding of non-photosynthetic vegetation under $15 \%$ in identifying closed forests. However, as this study indicates, the difference between closed forest and open forest in the Northern 
Sierra Madre is more subtle and refinement is necessary for higher classification accuracy.

\section{CONCLUSION}

In this paper, MESMA was applied to identify closed forest and open forest canopy in a hyperspectral image. This involved collecting endmember spectra, optimizing the spectral library and analysis of concurrence between two classified images. For the purpose of an initial assessment, MESMA of a hyperspectral image generally provides suitable results of classification between closed and open forests. However, for establishing baseline conditions for eventual monitoring of degradation in closed forests, field training data and additional information on the ground conditions of the study area is necessary. Also while hyperspectral imagery is a rich source of data, there is also the trade-off with its temporal availability, cost and processing requirement. Such issues need to be resolved when considering the monitoring of forest degradation through hyperspectral imagery. EO-1 was decommissioned in 2017 but a line-up of spaceborne hyperspectral sensors are in the pipeline. The launch of new sensors can possibly increase the temporal availability of hyperspectral imaging sensors.

\section{ACKNOWLEDGEMENTS}

The authors gratefully acknowledge the support of the program 'Digital Information for Monitoring and Evaluation' by the Department of Budget and Management.

\section{REFERENCES}

Biodiversity Management Bureau. (2015). Guidebook to Protected Areas of the Philippines. Quezon City, Philippines: Department of Environment and Natural Resources.

Bullock, E. L., Woodcock, C. E., \& Olofsson, P. (2018). Monitoring tropical forest degradation using spectral unmixing and Landsat time series analysis. Remote Sensing of Environment, (April), 0-1. https://doi.org/10.1016/j.rse.2018.11.011

Convention on Bilogical Divresity. (n.d.). Philippines - Country Profile. Retrieved from https://www.cbd.int/countries/profile/default.shtml?count $\mathrm{ry}=\mathrm{ph}$

Dennison, P. E., Halligan, K. Q., \& Roberts, D. A. (2004). A comparison of error metrics and constraints for multiple endmember spectral mixture analysis and spectral angle mapper. Remote Sensing of Environment, 93(3), 359-367. https://doi.org/10.1016/j.rse.2004.07.013

Dennison, P. E., \& Roberts, D. A. (2003). Endmember selection for multiple endmember spectral mixture analysis using endmember average RMSE. Remote Sensing of Environment, 87(2-3), 123-135. https://doi.org/10.1016/S0034-4257(03)00135-4

Deutsche Gesellschaft für Internationale Zusammenarbeit. (2012). Analysis of Key Drivers of Deforestation and Forest Degradation in the Philippines.

Food and Agricultural Organization. (2006). Forest degradation. Retrieved http://www.fao.org/3/j9345e/j9345e08.htm

Food and Agricultural Organization. (2015). No Title. Retrieved from http://www.fao.org/3/w7730e/w7730e0b.htm

Forest Management Bureau. (2019). Philippine Forests at a
Glance. Manila.

Kruse, F. A., Lekhoff, A. B., Boardman, J. W., Heidebrecht, K. B., Shapiro, A. T., Barloon, P. J., \& Goetz, A. F. H. (1993). The Spectral Image Processing System (SIPS)- Interactive Visualization and Analysis of Imaging Spectrometer Data. Remote Sensing of Environment, 44(145-163), 145-163.

Miettinen, J., Stibig, H., \& Achard, F. (2014). Remote sensing of forest degradation in Southeast Asia - Aiming for a regional view through $5-30 \mathrm{~m}$ satellite data. Global Ecology and Conservation, 2, 24-36. https://doi.org/10.1016/j.gecco.2014.07.007

Mitchell, A. L., Rosenqvist, A., \& Mora, B. (2017). Current remote sensing approaches to monitoring forest degradation in support of countries measurement , reporting and verification ( MRV) systems for REDD +. Carbon Balance and Management, 12(9), 22. https://doi.org/10.1186/s13021-017-0078-9

Plaza, A., Martínez, P., Pérez, R., \& Plaza, J. (2004). A Quantitative and Comparative Analysis of Endmember Extraction Algorithms From Hyperspectral Data, 42(3), 650-663.

Roberts, D. A., Gardner, M., Church, R., Ustin, S., \& Scheer, G. (1994). Mapping Chaparral in the Santa Monica Mountains Using Multiple Endmember Spectral Mixture Models, 4257(98).

Roth, K. L., Roberts, D. A., Dennison, P. E., Alonzo, M., Peterson, S. H., \& Beland, M. (2015). Differentiating plant species within and across diverse ecosystems with imaging spectroscopy. Remote Sensing of Environment, 167, 135-151. https://doi.org/10.1016/j.rse.2015.05.007

Somers, B., Asner, G. P., Tits, L., \& Coppin, P. (2011). Remote Sensing of Environment Endmember variability in Spectral Mixture Analysis : A review. Remote Sensing of Environment, 115(7), 1603-1616. https://doi.org/10.1016/j.rse.2011.03.003

Souza, C., Firestone, L., Moreira, L., \& Roberts, D. (2003). Mapping forest degradation in the Eastern Amazon from SPOT 4 through spectral mixture models, 87, 494-506. https://doi.org/10.1016/j.rse.2002.08.002

Tompkins, S., Mustard, J. F., \& Forsyth, D. W. (1997). Optimization of Endmembers for Spectral Mixture Analysis. Remote Sensing of Environment, 59(472-489), 18.

van der Meer, F. (1999). Iterative spectral unmixing ( ISU ). International Journal of Remote Sensing, 20(17), 34313436. https://doi.org/10.1080/014311699211462 